\title{
Problems of university-based scientists associated with clinical trials
}

\begin{abstract}
University faculty members who participate in clinical trials face a number of difficulties in connection with this association. Publication opportunities are often limited, and individual scholarship is difficult to express and evaluate within the context of a cooperative trial. Merit increases, promotion, and the award of tenure will usually require evidence of scholarly achievement outside the trial setting. For this reason, it seems inadvisable to recommend that a young investigator devote a major portion of his scholarly and research time to such an activity. A possible exception may be a full-time appointment for 1 to 2 years. Nonetheless, cooperative clinical trials are an important investigative tool and they should continue to be associated with academic centers. If appropriate administrative arrangements can be made, it should be possible to solve the academic problems of the young investigator associated with such trials.
\end{abstract}

Richard D. Remington, Ph.D. Ann Arbor, Mich. University of Michigan School of Public Health

I have been asked to discuss some of the problems faced by university faculty members who participate in clinical trials. I take this assignment to be limited to those problems directly related to the academic condition. Now, what is that academic condition? Faculty members entering an academic environment must engage in teaching, research, and service. They look forward to regular merit increases, to promotion, to the acquisition of academic tenure, and to the establishment of a professional and scientific reputation outside the institution in which they serve.

\section{Time commitments}

Many academic institutions will suggest an appropriate allocation of time to teaching, research, and service. The institution for which I am administratively responsible (the University

Reprint requests to: Dr. Richard Remington, Dean, School of Public Health, University of Michigan, Ann Arbor, MI 48109. of Michigan School of Public Health) suggests as a guideline that a faculty member should normally devote $40 \%$ of his time to teaching, $40 \%$ to research, and $20 \%$ to university, community, or professional service. Our school conducts a quantitatively oriented annual review designed to determine whether or not a given faculty member has performed at a level of quantity and quality consistent with established norms in each of these three areas. That evaluation is used to provide merit increases each year. A relatively elaborate system for promotion and the acquisition of tenure is in place within the school.

This system is based on review by tenured faculty within the department, and recommendation for promotion is then made to the Dean and Executive Committee. The recommendation and all documentation, including outside peer evaluation, are forwarded to an Advisory Committee on Academic Rank which then 
makes a recommendation to the Dean and Executive Committee for final transmission and ratification by the Board of Regents of the University.

Perhaps most academic institutions follow a roughly similar system. We encourage faculty members to participate in community service projects which will take them beyond their academic discipline as narrowly defined. We believe this is good for the school, for the university, and for the individual faculty member. After all, the development of an academic and scholarly reputation is important to any young scientist. We are not interested in establishing an indentured class of faculty members who are not in demand by other academic institutions. Furthermore, we believe that there exists a healthy degree of mobility and that not every faculty member will decide to remain within his or her department throughout a professional lifetime. Of course, we do not encourage mobility for mobility's sake alone.

\section{Participation in a clinical trial}

Now let us examine briefly the environment of the cooperative clinical trial. Whether within the context of a clinical center, a coordinating center, or a specialized data evaluation center such as a biochemistry laboratory or an electrocardiographic monitoring center, certain characteristics and requirements of a collaborative clinical trial are relevant to investigator participation. First, publication of results is ordinarily rigidly controlled by the apparatus of the trial, and there will be many more potential authors than publication opportunities. In a trial of reasonable duration a given young investigator will find it difficult to have his name individually associated with a large number of publications. Of course, rules vary and it may well be possible by policy for such investigators to list in their bibliographies all publications resulting from the collaborative trial with which they are associated. In some instances, however, this is not possible and in others a few scientists engaged in preparing an individual manuscript will be credited by name.

Second, the conduct of a collaborative research project requires adherence to a study design, protocol, and manual of operation. These documents will result from negotiations among senior scientists responsible for the initial design of the trial. This activity constitutes one of the greatest intellectual challenges within the trial as a whole. Thus, a young investigator must conform to a considerable degree to the restrictions implicit in the design and protocol. This produces a limitation on his ability to exercise independent intellectual judgment.

Against these problems, one must set the undeniable advantage to the faculty member of becoming acquainted with a large, cosmopolitan group of investigators located at a number of institutions. This arrangement can provide useful professional contacts and may even lead to future employment opportunities.

Reviewing groups and advisory boards have an important impact on the personal participation of academic investigators in clinical trials. Reviewers of contract and grant proposals will often expect individuals to devote a substantial amount of time to a collaborative trial. After all, the trial is important to the funding agency, to the reviewers who have devoted important time to providing an evaluation of the application, and to policy advisory boards and other groups who have made a similar time commitment. The reviewing groups can thus be expected to require a substantial investment of time from a number of investigators associated with the project.

At one institution where I served as Associate Dean for Research, it was our uniform practice in dealing with this latter problem to point out to funding agencies and reviewing bodies that, by school policy, a faculty member was ordinarily permitted to spend up to $40 \%$ of his time in research. Thus a $20 \%$ total time commitment amounted to a $50 \%$ total research obligation. This institution's record at securing funding for its grants and contracts was good, and I am unaware that this policy ever resulted in a disapproval or a low priority rating of one of our applications. I believe the forthright statement of institutional policy as a part of each application may have been helpful in this connection.

How should we, as senior professors and administrators, advise our young faculty colleagues concerning their potential participation in cooperative clinical trials? On the one hand, 
the opportunity to associate with colleagues in comparable institutions is undeniably valuable. The excitement of being engaged in important public research which can have a major influence on therapeutic practice is considerable. The excitement of participating in the group process that leads to collective decisions is stimulating and informative. The experience gained in associating with a collaborative project is inherently maturing; yet these benefits are not without cost. Promotions committees, executive committees, and deans expect to see evidence of a young investigator's intellectual independence and maturity before they recommend promotion or appointment to a tenured position. Occasionally, affiliation with a cooperative trial can provide such evidence. More often, however, even the participants in such a trial will have difficulty providing an objective assessment of the independent, individual contribution of a given person. Group processes are inherently complex, and interactions are often as important in determining outcomes as individual actions.

Occasionally, a young investigator will be able to affiliate himself with an ancillary or spin-off project away from the main line of investigation. In this event, he may be able to receive substantial promotion points for that activity. More often, however, it will be difficult for a young faculty member to receive appropriate credit for the massive investment of time required in the conduct of a cooperative trial. Thus, it seems to me that we have an obligation to warn the potential young collaborator who is about to affiliate with a clinical trial that he can simply not expect full reward for such participation in terms of promotion, tenure, and the like. My advice is, therefore, that young investigators should not commit a major fraction of their investigative time to a cooperative clinical trial. Certainly, I am very wary of assigning a young person a full-time responsibility to such an effort. An exception may be the full-time assignment of an individual for a year or two, with the understanding that such experience may well be consistent with the individual's ultimate career goals and that he will, after the experience with the cooperative trial, move on to other activities in which such a major time commitment to such a relatively unrewarding environment will not be necessary.

\section{Possible accommodations}

Do such personnel difficulties constitute insurmountable barriers to the success of a cooperative clinical trial, and do they produce irreconcilable differences? I think not. I believe we should not expect young academics to devote over many years most of their time to clinical trials. We should encourage them to engage in independent scholarly activity, more likely to contribute to academic advancement. Should we attempt to alter promotion and tenure policies in our academic institutions? We can do so if we wish, but I suspect that, at least in the major research universities, we will not often be successful. Furthermore, I must question the values intrinsic in such an effort. Independent scholarship is still valuable, although I am personally a believer in collaborative research. An analysis we conducted of authorship practices in a wide variety of scientific journals a few years ago suggests an increasing trend, even in the so-called basic sciences, toward multiauthored papers and away from single authorship. Our practice at Michigan is to count coauthorships at full weight with solo authorships, at least as far as merit increases are concerned. I suspect that promotion and tenure committees, on the other hand, will continue to place some emphasis on individually authored contributions.

Does this mean that academic and scientific staffs associated with cooperative clinical trials should be drawn either from nonacademic institutions or from nonresearch-oriented universities? It does not. I personally believe that, with appropriate compromises between the management of the trial and the management of the institution, an accommodation can almost always be effected. As an administrator, however, I operate by the principle that the academic administration must take pains to avoid entrapment of young faculty members. (The administration should help young faculty avoid entrapment by others, as well.) On the contrary, the academic administrator should accept the challenge of urging senior faculty to contribute to the development of junior academic colleagues. The administrator should 
seek an environment in which reasonable career goals can be attained by assistant professors and instructors. This encouragement of junior members of the faculty clearly benefits the institution and also the individual faculty member.

I am aware of the fact that some academic departments have devoted a substantial fraction of their total effort to collaborative research. I believe that this arrangement can be suitable, provided that this policy does not work a disservice to junior faculty. Indeed, the presence of an active collaborative trial or its coordinating center in the academic department can create an environment in which opportunities for independent scholarship will be promoted rather than inhibited. This seems particularly true in methodologic areas such as epidemiology and biostatistics. Certainly, there is no shortage of challenging design, management, and analytic problems arising from collaborative research that can require the development of new methodology.

Finally, I believe we should all pool our thinking and attempt to develop innovative solutions to the problems faced by the young investigator in collaborative research. Incidentally, although I have focused on the young, these problems exist in one form or another for investigators of all ages. I believe that with a large dose of good will and some incisive thinking we can fund, organize, and administer cooperative trials in a manner that gives increased attention to the academic problems faced by the university investigator affiliated with a long-term cooperative project. 